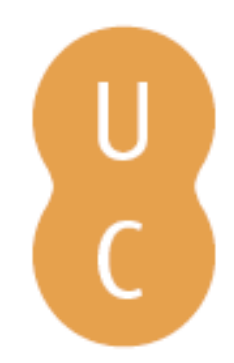

\title{
nommalina
}

\section{Navegação indoor baseada na rede WiFi como suporte a serviços baseados na localização: estudo de caso no Campus da UL}

Autor(es): $\quad$ Simões, Diogo; Catalão, João

Publicado por: Imprensa da Universidade de Coimbra

URL

persistente: URI:http://hdl.handle.net/10316.2/37074

DOI: $\quad$ DOI:http://dx.doi.org/10.14195/978-989-26-0983-6_21

Accessed : $\quad$ 26-Apr-2023 15:12:29

A navegação consulta e descarregamento dos títulos inseridos nas Bibliotecas Digitais UC Digitalis, UC Pombalina e UC Impactum, pressupõem a aceitação plena e sem reservas dos Termos e Condições de Uso destas Bibliotecas Digitais, disponíveis em https://digitalis.uc.pt/pt-pt/termos.

Conforme exposto nos referidos Termos e Condições de Uso, o descarregamento de títulos de acesso restrito requer uma licença válida de autorização devendo o utilizador aceder ao(s) documento(s) a partir de um endereço de IP da instituição detentora da supramencionada licença.

Ao utilizador é apenas permitido o descarregamento para uso pessoal, pelo que o emprego do(s) título(s) descarregado(s) para outro fim, designadamente comercial, carece de autorização do respetivo autor ou editor da obra.

Na medida em que todas as obras da UC Digitalis se encontram protegidas pelo Código do Direito de Autor e Direitos Conexos e demais legislação aplicável, toda a cópia, parcial ou total, deste documento, nos casos em que é legalmente admitida, deverá conter ou fazer-se acompanhar por este aviso.

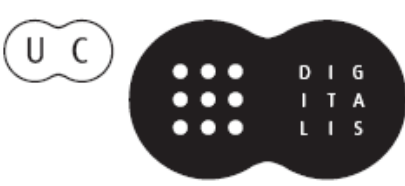




\section{$\forall$ \\ TAS DAS I JORNADAS LUSÓFONAS DE CIÊNCIAS E TECNOLOGIAS DE INFORMAÇÃO GEOGRÁFICA}

Editores

José Gomes dos Santos

Cidália Fonte

Rui Ferreira de Figueiredo

Alberto Cardoso

Gil Gonçalves

José Paulo Almeida

Sara Baptista 


\begin{abstract}
ARTIGO 21
NAVEGAÇÃO INDOOR BASEADA NA REDE WIFI COMO SUPORTE A SERVIÇOS BASEADOS NA LOCALIZAÇÃO: ESTUDO DE CASO NO CAMPUS DA UL
\end{abstract}

SIMÕES, Diogo ${ }^{1}$ \& CATALÃO, João ${ }^{2}$

\begin{abstract}
${ }^{1}$ Departamento de Engenharia Geográfica, Geofísica e Energia - DEGGE; Faculdade de Ciências da Universidade de Lisboa (Portugal); Campo Grande, 1749-016 Lisboa, Portugal; email: diogo.m.simoes@gmail.com 2 Departamento de Engenharia Geográfica, Geofísica e Energia - DEGGE ; Faculdade de Ciências da Universidade de Lisboa (Portugal); Instituto D. Luiz (Portugal); Campo Grande, 1749-016 Lisboa, Portugal; Tel: +351 217500833; email: jcatalao@fc.ul.pt
\end{abstract}

\title{
RESUMO
}

Os serviços baseados na localização dos utilizadores têm tido uma grande evolução ao longo destes últimos anos. O novo desafio prende-se com a possibilidade desses serviços serem transportados de um ambiente outdoor para indoor. Variados algoritmos e técnicas de posicionamento foram desenvolvidos, através de diversas tecnologias para o cálculo de posição dos dispositivos. Alguns destes sistemas já se encontram instalados em centros de grande afluência, como os centros comerciais. Neste artigo vamos apresentar uma solução de um serviço baseado na localização em ambientes fechados, baseado na rede WiFi, para o caso do campus da Universidade de Lisboa. O trabalho desenvolvido pretende demonstrar o potencial de várias tecnologias combinadas, tendo como resultado uma aplicação móvel, desenvolvida na linguagem Android, desempenhando um serviço baseado na localização do utilizador num espaço indoor. 


\title{
PALAVRAS-ChAVE
}

Serviços baseados na localização, Tecnologia WiFi, Android, Aplicações móveis, Técnicas de posicionamento indoor.

\section{INDOOR LOCATION-BASED SERVICES:}

\section{A CASE OF STUDY ON CAMPUS OF UL}

\begin{abstract}
Location-based services had a great evolution over the last few years. The new challenge lies in the possibility of these services being transported to a billboard for an indoor environment. Various algorithms and positioning techniques were developed, involving various technologies for calculating the position of the device. Some of these systems are already installed in places of heavy traffic, such as shopping centers. In this article we present a solution based on a location indoors service based on WiFi network, for the case of the University of Lisbon campus. The work aims to demonstrate the potential of several technologies combined resulting in a mobile application. This application will be developed in Android language, performing a process based on user indoor location.
\end{abstract}

\section{KEYWORDS}

Location-based services, WiFi network, Android, Mobile applications, Indoor positioning.

\section{INTRODUÇÃO}

O desenvolvimento da cidade como elemento focal da informação para o negócio, governança e da organização social tem promovido a concentração de pessoas e bens na sua área de influência maximizando as relações entre espaço e informação. O crescimento das cidades, quer em número e tamanho, em todo o mundo é o testemunho da sua maturidade como processo dominante da organização social e económica. Em oposição ao determinismo tecnológico que previa uma dispersão da cidade no espaço, com o evoluir das tecnologias de comunicação potenciando o teletrabalho, a coevolução do espaço geográfico e eletrónico preconiza o desenvolvimento da cidade em simbiose com o crescimento das redes de telecomunicação e da aceleração das taxas de transmissão.

As cidades vão redefinindo a sua forma devido às relações comple- 
xas entre capital, tecnologia e espaço [1]. Estas que beneficiaram de investimentos nos acessos físicos são agora beneficiadas com excelentes infraestruturas de TIC (Tecnologias da Informação e Comunicação) e são centros de conteúdos de Internet porque é na cidade que se foi acumulando o conhecimento. Neste contexto, as cidades evoluíram no sentido da concentração de serviços e pessoas com o crescimento da construção em altura como forma de suprir a dispersão no espaço favorecendo a concentração de espaços multivalências: comerciais, lazer e trabalho. Estima-se que na atualidade mais de $80 \%$ do nosso tempo é passado dentro de edifícios. Esta nova realidade representa uma oportunidade para novos negócios baseados na localização das pessoas. De facto, a concentração massiva de pessoas em espaços comuns, aleada a uma melhoria significativa das redes de comunicação, com cada vez maior largura de banda, possibilitam uma vasta gama de serviços entre os quais os serviços baseados na localização.

A localização fora de ambientes fechados, através do Sistema de Posicionamento Global, é utilizada hoje para as mais variadíssimas áreas, mais críticas como a aviação ou no auxílio de resgates, em áreas com um teor mais turístico, com um caracter mais desportivo ou mesmo até com um cariz social, como o exemplo das redes sociais. Contudo o sistema referido acima tem as suas limitações no que toca a ambientes fechados em que o sinal, que permitirá calcular a localização de um dispositivo, não chega nas melhores condições. Para combater esta limitação foram desenvolvidos diversos algoritmos baseados nas mais variadas tecnologias [2], como o Infravermelho, RFID (Radio-Frequancy Identification), WLAN (Wireless Local Area Network), Bluetooth, UWB (Ultra Wide Band), entre outros.

Nos dias de hoje, a tecnologia WLAN é a mais usada por não requerer investimento em novas infraestruturas, mas sobretudo por estar disponível em dispositivos das últimas gerações da comunicação móvel. Estes dispositivos estão cada vez mais presentes no quotidiano das pessoas, sendo considerado em muitos casos imprescindível, devido às suas mais variadas utilizações.

No entanto, a tecnologia WLAN tem algumas limitações, sobretudo em 
espaços fechados, devido à complexidade da construção dos edifícios, com todos os elementos presentes a representarem um obstáculo na propagação do sinal Wifi. Tudo isto, aleado a uma deficiente distribuição dos pontos de acesso, ao longo do edifício, pode revelar-se uma dificuldade na estimação da localização do dispositivo. Para combater algumas destas limitações, são usadas diversas técnicas matemáticas, como no caso do algoritmo de posicionamento indoor baseado na rede Wifi [3], que através de uma das técnicas de Trilateração, utilizando a força do sinal, para estimar a localização do dispositivo, envolve posteriormente o filtro de Kalman e o Método do Mínimo dos Quadrados que permite melhorar a precisão da localização do dispositivo móvel. Existem ainda outras técnicas de localização em ambientes fechados [2] [4] [5], como a Triangulação, por análise de todo o ambiente envolvente e por Proximidade. A técnica de análise de todo o ambiente envolvente é também conhecida por utilizar o processo de Fingerprinting [6], em que o dispositivo reconhece pontos específicos do ambiente em que se encontra e assim estima a sua localização. Em sistemas que usam esta técnica existe uma base de dados em que são guardados pontos de localização recolhidos a priori. Aquando do cálculo das coordenadas da localização do dispositivo, é estimado a mesma, com base nos pontos recolhidos anteriormente.

Este documento será dividido por tópicos e subtópicos. No primeiro tópico será apresentado e abordado o tema, sendo explicitados todos os detalhes do mesmo. No segundo tópico será apresentado todas componentes e características do sistema, sendo este dividido em componentes, correspondendo a subtópicos. Por último e com o título de Discussão e Conclusão serão discutidos resultados e as respetivas conclusões.

\section{APRESENTAÇÃo DO TEMA}

A massificação do uso de dispositivos móveis e a sua evolução, no que toca à comunicação com dispositivos não móveis potenciou o desenvolvimento de novas formas de negócio baseadas na mobilidade, como os serviços baseados na localização (Location Based Services).

Como tal, e para dar resposta ao problema apresentado na secção 
anterior, decidimos criar uma aplicação capaz de dar auxílio à navegação indoor e outdoor por parte de utilizadores, através dos seus dispositivos móveis. Esta cruzará várias tecnologias existentes, com o intuito de podermos analisar todo o processo, encontrando assim soluções mais eficientes no que toca à orientação e posicionamento dos utilizadores em ambientes fechados.

Como parte integrante deste grupo de utilizadores estarão certamente turistas que necessitem de auxílio na navegação dentro de um museu ou mesmo num aeroporto ou então, estudantes que necessitem de se deslocar dentro de um campus de uma universidade. Outra parte dos utilizadores que poderão usufruir da aplicação serão todos os clientes que desejem fazer compras num supermercado, ou num centro comercial, onde serão guiados até aos locais ou produtos desejados.

Todo este trabalho centrou-se no contexto da deslocação indoor e outdoor de um aluno que desconhece o campus ou mesmo um professor visitante, que pode necessitar o fornecimento de alguns pontos de interesse dentro do campus da Faculdade de Ciências da Universidade de Lisboa. Este serviço pode consistir, também, no aviso de proximidade ou na comunicação de informação contextualizada (como eventos, menus de refeições, etc).

\section{METODOLOGIA UTILIZAdA}

Nos subtópicos abaixo será dado uma visão de todas as ferramentas e tecnologias que foram utilizadas no decorrer do trabalho efetuado.

\subsection{Ferramentas Utilizadas}

Para a elaboração de todo o sistema foi necessário a utilização de um conjunto de ferramentas tanto a nível de Software, como a nível de Hardware. Para enumerar as ferramentas utilizadas vamos dividir todo o Sistema em três grandes componentes, o Cliente - Aplicação móvel, o Servidor e a Base de Dados.

Primeiramente toda a componente do Cliente - Aplicação foi desenvolvida no ambiente da linguagem de programação Java para poder 
correr em dispositivos com Sistema Operativo Android, sendo todo o seu código elaborado e compilado através do Software Eclipse IDE for Java Developers, com a versão Helios Service Release 2. Os dispositivos móveis usados para testar a aplicação foram o tablet Samsung Galaxy Tab 10 e o smartphone LG Google Nexus 5.

Toda a componente do Servidor foi desenvolvida num ambiente da linguagem de programação Java SE 1.7, sendo elaborado e compilado o seu código através do Software Eclipse IDE for Java Developers, com a versão Helios Service Release 2.

Para a disponibilização dos mapas para a interface da aplicação foi utilizado o software ArcGIS Server, instalado numa máquina com IP público.

Por fim a componente da Base de Dados foi criada através do Software PostGreSQL 9.2 com extensão PostGIS 2.O.

Tanto a componente referente à Base de Dados, como a componente do Servidor, tiveram como base de Hardware um computador incorporado com as seguintes características, um processador Intel Core i3 2120 CPU $3.30 \mathrm{GHz}$, uma memória RAM de $4 \mathrm{~Gb}$ e com o sistema operativo Windows 7 Professional 32-Bit.

\subsection{Infraestrutura do espaço de estudo}

À semelhança do que acontece com a maioria de todos os campus universitários, o edifício C8, da Faculdade de Ciências da Universidade de Lisboa, está equipado com um conjunto de Pontos de Acesso (AP), espalhados por todo o edifício, de modo a cobrir a maior parte de todos os locais do edifício, como mostra a Figura 1.

Estes dispositivos, AP, permitem a interconexão entre dispositivos móveis, possibilitando o acesso à rede da Universidade e consequentemente à rede da Internet. A interconexão com os dispositivos móveis é efetuada com recurso à tecnologia WiFi, que permite que os dispositivos se conectem, entre si, sem o recurso a cabos. Para que todos os dispositivos móveis se possam conectar aos AP espalhados pelo edifício é necessário que captem as ondas eletromagnéticas emitidas por cada AP. Cada dispositivo móvel está, segundo intervalos de tempo predefinidos, a 
medir a intensidade do sinal de todos os AP que recebe, avaliando assim o melhor ponto de acesso para se conectar.

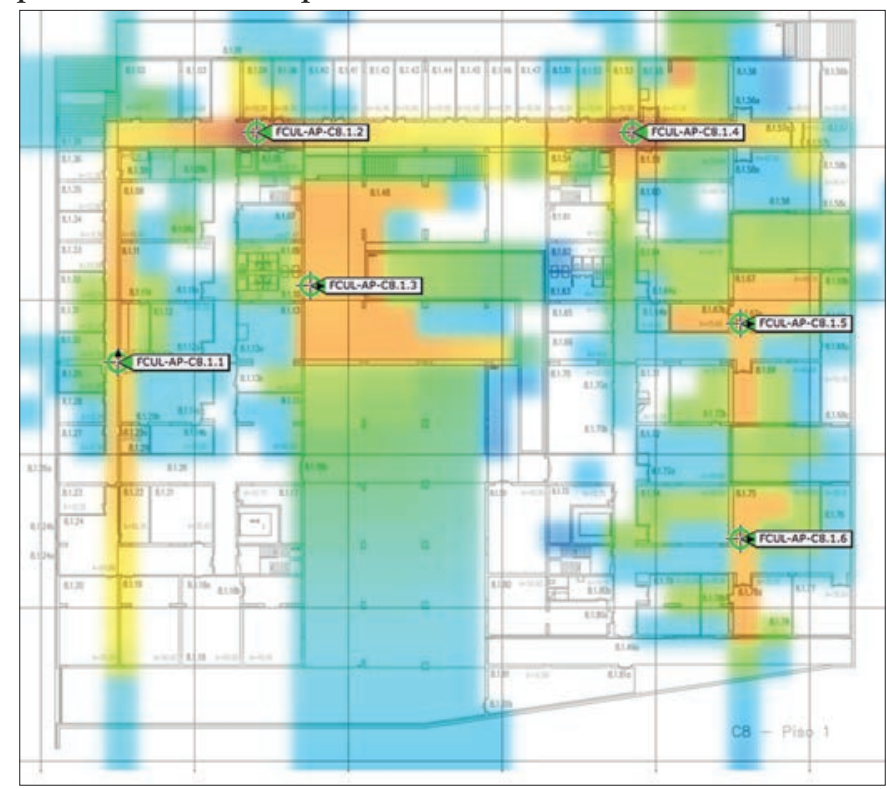

Figura 1 - Modelo de propagação do sinal WiFi no edifício do C8

Todos os AP são identificados com um identificador único, o $M A C$ Address, que permite univocamente identificar a qual dos AP, qualquer dispositivo móvel está conectado. Para um utilizador comum este identificador não será relevante, no entanto, no contexto do sistema a criar vai ser de extrema importância. Esta informação torna-se crucial para o sistema uma vez que vai servir de identificador na Base de Dados, onde estão guardados todos os MAC Address, de cada AP. Cada entrada na Base de Dados relativa a cada um dos AP terá associado um par de coordenadas, Latitude e Longitude, que permitirá identificar o local da instalação do AP.

\subsection{Arquitetura do Sistema}

Como é mostrado na Figura 2, todo o Sistema é dividido em três componentes distintas, a componente do Cliente, do Servidor e da Base de Dados. 


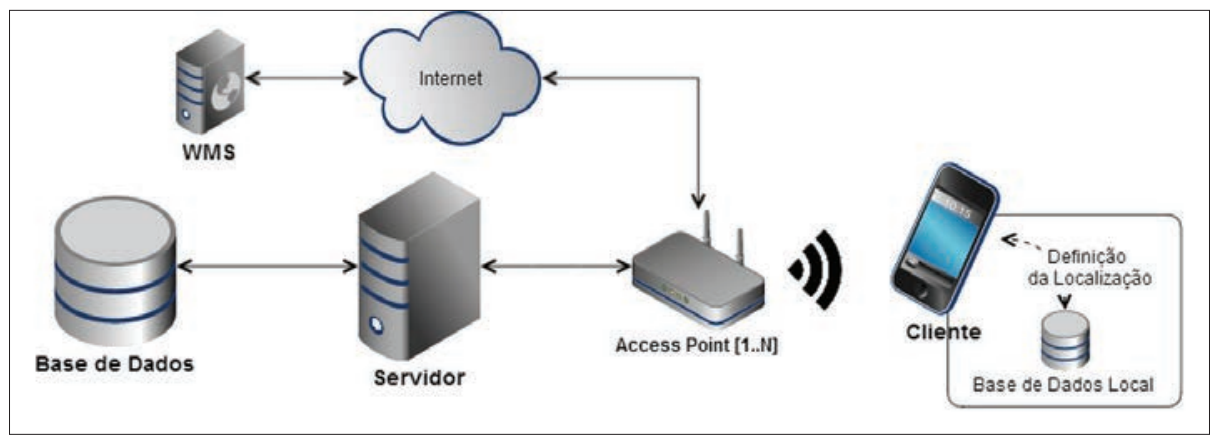

Figura 2 - Modelo de Arquitetura do Sistema

\subsection{Componentes do Sistema}

Neste subtópico serão explicadas cada componente do sistema.

\subsubsection{Cliente Aplicação}

A componente do Cliente é constituída por uma aplicação móvel, que permite aos utilizadores poderem usufruir de todo o Sistema de Navegação e por uma Base de Dados Local, disponibilizada através de uma biblioteca existente para o Android, chamada SQLite.

Esta biblioteca é responsável por todas as operações de escrita e leitura efetuadas na Base de Dados criada e presente no disco do dispositivo.

Como explicado, na secção anterior, todos os AP terão de estar armazenados numa base de dados com informação acerca do seu identificador, MAC Address e informação da sua localização. A tecnologia do SQLite vai permitir que toda essa informação possa ser armazenada no disco do dispositivo, para que assim possa ser evitada uma chamada à Base de Dados do Servidor. Todo este processo vai tornar mais eficiente a execução da aplicação.

A aplicação móvel tem como interface um mapa, do edifício, que é disponibilizado através do formato Web Mapping Service (WMS), presente no software ArcGIS Server. Toda esta interação será explicada no subtópico referente à componente do Servidor.

O cálculo da posição do utilizador será efetuada, também, na componente do Cliente. Este cálculo terá diferentes variáveis que foram 
calculadas e definidas com base em experiências efetuadas ao longo do processo de desenvolvimento.

Para o cálculo da posição do utilizador foi necessário estabelecer uma relação entre a intensidade do sinal recebido no dispositivo e a distância do mesmo ao AP, a que está ligado.

Esta relação pode ser traduzida através de um modelo Power Law [7], enunciado na página 3, do artigo referenciado, através a equação seguinte:

$$
\text { RSSI }[\mathrm{dBm}]=-10 n \log 10(\mathrm{~d})+\mathrm{A}[\mathrm{dBm}]
$$

Onde, $\mathrm{n}$ é a variável que representa a perda de intensidade do sinal proveniente dos AP, A é a intensidade de sinal medido no dispositivo a 1 metro do AP, RSSI é o valor de intensidade medido no momento do cálculo e d é a distância estimada do dispositivo ao AP.

Com base na equação 1 a distância é calculada, resolvendo a equação em ordem a d, obtendo-se assim a distância a que o dispositivo está do AP.

Com a distância, entre o dispositivo e o AP, definida, através da equação 1, é necessário calcular as coordenadas exatas do utilizador. Para isso teremos de, às coordenadas do AP, somar a distância deste ao utilizador.

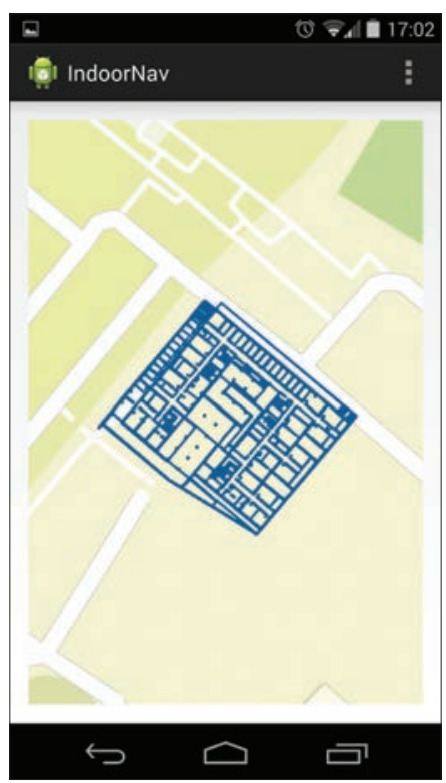

Figura 3 - Figura de uma dos ecrãs da aplicação, com as layers obtidas através de WMS 


\subsubsection{Servidor}

Como em todos os sistemas do tipo Cliente - Servidor, a componente do Servidor reserva um papel muito importante para o bom funcionamento do sistema. Esta componente tem o papel de estabelecer comunicação com a componente do Cliente, a aplicação móvel, processar todos os pedidos vindos do mesmo e ainda estabelecer comunicação com a componente da Base de Dados.

Nesta parte do sistema está incluído um serviço que é prestado através do Software ArcGIS Server. Este serviço será responsável por disponibilizar, no formato de imagem de todos os mapas, tanto do exterior como do interior do edifício. Estes são disponibilizados através da especificação do formato WMS. O WMS é uma especificação de formato que fornece uma interface (HyperText Transfer Protocol) HTTP simples para solicitar imagens de mapas. Cada pedido WMS define uma layer geográfica e uma área de interesse para ser processado. Como resposta ao pedido é enviado uma ou mais imagens, no formato de JPEG, PNG, etc. Estas imagens vão ser interpretadas e exibidas por um navegador web ou então, como no caso, por uma aplicação Android. Podem ser solicitadas várias layers, sobrepondo-se assim umas às outras, aquando a exibição das mesmas.

\subsubsection{Base de Dados}

Para que o utilizador pudesse obter informação sobre as áreas e os espaços que o rodeavam, foi necessário criar uma Base de Dados com toda a informação dos pontos de interesse presentes no edifício do objeto de estudo.

Toda a informação presente na tabela da Base de Dados foi carregada através do programa PostGIS 2.O Shapefile and DBF Loader que permite, através de um ficheiro do tipo shapefile, carregar dados para uma tabela previamente criada. A tabela será constituída pelos campos integrados no ficheiro shapefile.

A Base de Dados criada para armazenar os pontos de interesse, ao longo do edifício, é constituída apenas por uma tabela. Esta é constituída por campos que caracterizam os pontos de interesse, como por exemplo, as suas coordenadas e a descrição do local. 


\subsection{Funcionamento do Sistema}

Aquando da iniciação da aplicação é criada uma ligação entre a componente do Cliente e o programa do Servidor para que possam ser transferidos dados entre estas duas componentes. O canal desta ligação permanece sempre aberto enquanto a aplicação estiver em execução.

Em simultâneo é criada uma tarefa de examinação de todos os Pontos de Acesso presentes no raio de cobertura do dispositivo, para que se possa ser identificado o AP a que o dispositivo está conectado. Após essa análise é identificado o AP, através da intensidade da força do sinal que é recolhido pelo dispositivo. Através das propriedades do AP selecionado, será recolhido o $M A C$ Address do mesmo, que posteriormente será enviado para a Base de Dados Local. Esta transação será finalizada com a obtenção das coordenadas do AP correspondente ao MAC Address selecionado. Estas coordenadas e a distância calculada, através da intensidade do sinal que chega ao dispositivo, serão utilizadas para calcular a posição geográfica do utilizador.

Enquanto o ponto da localização do utilizador é mostrado no ecrã do dispositivo, está em curso uma tarefa que terá como objetivo obter todos os pontos de interesse perto da localização do utilizador.

A ligação criada com o Servidor, aquando da iniciação da aplicação, é agora utilizada para enviar as coordenadas da localização do utilizador. Estas ao chegarem à componente do Servidor são embebidas numa query SQL (Structured Query Language), previamente criada, para que esta possa ser interpretada pela Base de Dados que contém todos os Pontos de Interesse. De referir, que a Base de Dados criada e instalada no computador do Servidor está em constante funcionamento, o que permite à componente do Servidor criar ligações quando existir dados de Pontos de Interesse a serem recolhidos.

A Base de Dados ao processar todo o pedido efetuado, devolverá informação acerca dos Pontos de Interesse selecionados. Essa informação incluirá as coordenadas do ponto, bem como a descrição do mesmo. Os Pontos de Interesse irão estar dentro de um raio (em metros) definido na query efetuada pelo Servidor.

Após a receção da informação por parte do Servidor, este envia no- 
vamente os resultados dos Pontos de Interesse, para a aplicação, que interpreta todos os dados e como mostra a Figura 4, exibe-os no ecrã sobre a forma de icons no mapa.

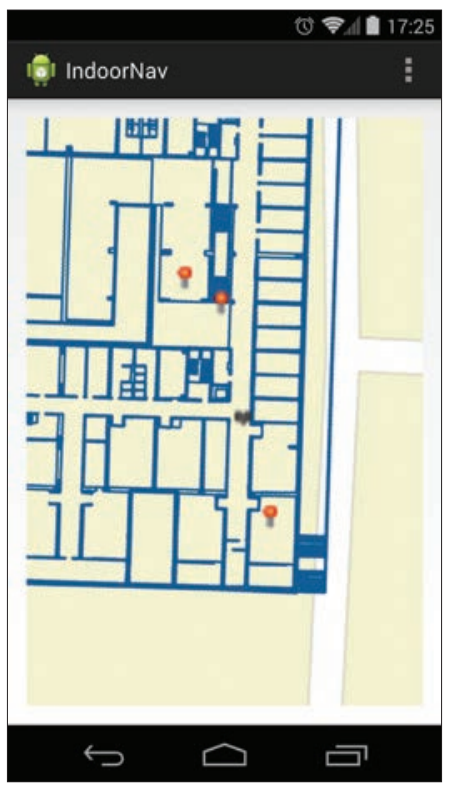

Figura 4 - Figura com os ícons dos pontos de interesse, bem como a localização do dispositivo

Através da imagem acima ilustrada, podemos verificar que com o ícon a preto temos a localização do utilizador e com os icons a vermelho os pontos de interesse.

\section{DiscuSSÃo DE RESUlTAdos}

Com este modelo de arquitetura, podemos perceber que neste momento todas as alterações que possam existir na componente do Cliente, não irão afetar o funcionamento de todo o Sistema. Esta abstração foi possível com a base de dados instalada no dispositivo. Deste modo, a informação referente à localização dos AP do edifício, armazenada nessa base de dados é estática, ou seja não irá sofrer mudanças. Uma das razões para que a informação referente aos pontos de interesse não seja armazenada na base de dados do dispositivo é que todos estes pontos têm uma grande probabilidade de serem alterados. Isto poderá acontecer num contexto 
em que os pontos de interesse sejam salas de aula em que ano após ano há uma remodelação das aulas lecionadas em cada sala.

\section{CONCLUSÃo}

Um dos grandes objetivos deste trabalho consistia na integração de diferentes tecnologias e ferramentas no desenho de uma solução eficiente para a navegação indoor. Como podemos verificar, no subtópico do Funcionamento do Sistema, conseguimos relacionar todas as tecnologias e obter assim um resultado satisfatório.

$\mathrm{Na}$ aplicação desenvolvida evidenciou-se o potencial da integração de sistemas de posicionamento baseados na rede $\mathrm{WiFi}$, a consulta em mobilidade de serviços WMS e da interação dos dispositivos móveis com bases de dados espaciais para fornecimento de serviços baseados na localização em tempo real.

\section{BIBLIOGRAFIA}

GRAHAM, Stephen \& Marvin, Simon (2001) - "Splintering Urbanism", Routledge, London.

HIGHTOWER, Jeffrey \& Borriello, Gaetano (2001) - "Location Sensing Techniques", IEEE Computer, Junho 2001.

ABOODI, Ahed \& Tat-Chee Wan (2012) - "Evaluation of WiFi-based Indoor (WBI) Positioning Algorithm", in Mobile, Ubiquitous, and Intelligent Computing (MUSIC), 2012 Third FTRA International Conference, Vancouver, Canadá, 26-28 June 2012, 260264.

BALLAZHI, Rilind - "Wireless Indoor Positioning Techniques", in Communication Systems V, B. Stiller et al. (Eds.), Zürich, Switzerland, 5468

HUI, Liu \& Houshang, Darabi \& Banerjee, Pat \& Jing, Liu (2007) - "Survey of Wireless Indoor Positioning Techniques and Systems”, in Systems, Man, and Cybernetics, Part C: Applications and Reviews, IEEE Transactions on Volume 37, Issue 6, Nov. 2007, 1067 - 1080

LANDU JIANG (2012) - "A WLAN Fingerprinting Based Indoor Localization Technique”, Lincoln, Nebraska, Julho, Dissertação apresentada à Universidade de Nebraska Lincoln

OGUEJIOFOR O.S., Okorogu V.N., Adewale Abe, Osuesu B.O (2013) - "Outdoor Localization System Using RSSI Measurement of Wireless Sensor Network", in International Journal of Innovative Technology and Exploring Engineering (IJITEE), Volume-2, Issue-2. 
Série Documentos

Imprensa da Universidade de Coimbra

Coimbra University Press

2015

- U M

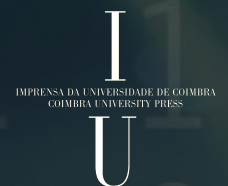

